\title{
An Optimization Method Combining RSSI and PDR Data to Estimate Distance Between Smart Devices
}

This paper was downloaded from TechRxiv (https://www.techrxiv.org).

\section{LICENSE}

CC BY-NC-SA 4.0

SUBMISSION DATE / POSTED DATE

$25-12-2020$ / 31-12-2020

CITATION

Zhao, Bo; Zheng, Chao; Ren, Xinxin; Dai, Jianrong (2020): An Optimization Method Combining RSSI and PDR Data to Estimate Distance Between Smart Devices. TechRxiv. Preprint.

https://doi.org/10.36227/techrxiv.13488738.v1

$\mathrm{DOI}$

10.36227/techrxiv.13488738.v1 


\title{
An Optimization Method Combining RSSI and PDR Data to Estimate Distance Between Smart Devices
}

\author{
Bo Zhao, Chao Zheng, Xinxin Ren, and Jianrong Dai
}

\begin{abstract}
Distance estimation methods arise in many applications, such as indoor positioning and Covid-19 contact tracing. The received signal strength indicator (RSSI) is favored in distance estimation. However, the accuracy is not satisfactory due to the signal fluctuation. Besides, the RSSI-only method has a large ranging error because it uses fixed parameters of the path loss model. Here, we propose an optimization method combining RSSI and pedestrian dead reckoning (PDR) data to estimate the distance between smart devices. The PDR may provide the high accuracy of walking distance and direction, which is used to compensate for the effects of interference on the RSSI. Moreover, the parameters of the path loss model are optimized to dynamically fit to the complex electromagnetic environment. The proposed method is evaluated in outdoor and indoor environments and is also compared with the RSSI-only method. The results show that the mean absolute error is reduced up to $0.51 \mathrm{~m}$ and $1.02 \mathrm{~m}$, with the improvement of $10.60 \%$ and $64.55 \%$ for outdoor and indoor environments, respectively, in comparison with the RSSI-only method. Consequently, the proposed optimization method has better accuracy of distance estimation than the RSSI-only method, and its feasibility is demonstrated through real-world evaluations.
\end{abstract}

Index Terms-Distance estimation, optimization method, received signal strength indicator, pedestrian dead reckoning, Covid-19

\section{INTRODUCTION}

$\mathrm{I}^{\mathrm{N}}$ $\mathrm{N}$ the field of the Internet of things (IoT), excellent distance estimation is the key point for many applications [1], such as indoor positioning for wireless sensor networks (WSNs) and danger alerts for unmanned vehicles on the road [2], [3]. For human beings, currently, mobile smartphone apps are used to facilitate Covid-19 contact tracing [4], [5]. If two people carrying smartphones contact close to one another (generally, within 2 meters), then the apps on their smartphones will both record this contact event. The exposure notifications will be provided when one is diagnosed with Covid-19. The apps focus on the task of proximity sensing, which is relied on predicting accurate distance between the two smartphones [6].

Bo Zhao is with the Department of Engineering Physics, Tsinghua University, Beijing 100084, China; and the Key Laboratory of Particle \& Radiation Imaging, Ministry of Education (Tsinghua University), Beijing 100084, China (e-mail: paulzhao@mail.tsinghua.edu.cn).

Chao Zheng and Xinxin Ren are with the Shenzhen Haichuang Era Medical Technology, Shenzhen 518000, China (e-mail: zhengchao@hcsd-med.com; renxinxin@hcsd-med.com).
In recent years, the received signal strength indicator (RSSI) of Bluetooth low-energy (BLE) has been exploited for distance estimation [7], [8]. RSSI distance estimation method is based on the theoretical log-distance path loss (LDPL) model, which describes that the signal value attenuates as the distance increases [9], [10]. The LDPL model contains two main parameters, the RSSI at the reference distance (named as $A$ ) and the path loss exponent (named as $n$ ) [11]. These two parameters need to be calibrated before distance measurement for a known scenario. However, for an unknown situation, distance estimation may have inaccuracies because it uses parameters not fitted to this environment [12]. Furthermore, RSSI fluctuates significantly due to electromagnetic interference, especially in an indoor environment [13]. Despite the uncertainties in RSSI and LDPL model, the RSSI-only method is still a widely used tool for distance estimation applications, such as Google/Apple Exposure Notification (GAEN) app for Covid-19 contact tracing [14]. Douglas J. Leith and Stephen Farrell report on the evaluation results of this app in a commuter bus [15]. They find that the attenuation level indicated by the app need not increase with the distance between phones.

On the other hand, pedestrian dead reckoning (PDR) is recognized as a relative localization technique, which predicts the current location by taking into account the three main inputs, i.e. pedestrian's start position, walking distance, and walking direction [16]. It utilizes multiple sensors available in smart devices; for instance, magnetometer, gyro, and accelerometer sensors [17], [18]. Since the properties of the RSSI-based distance estimation method and PDR-based localization method are complementary, so the combination of these two methods would be beneficial to improve the estimation accuracy [19]. Moreover, recent studies try to dynamically adjust the parameters of the RSSI-distance model by using a neural network. Shi et al and Li et al built the RSSI-distance model with the backpropagation neural network (BPNN), which requires a huge amount of data to train the neural network before distance estimation [20], [21].

Given the need for better distance estimation to compensate

Corresponding author: Jianrong Dai is with the National Cancer Center/National Clinical Research Center for Cancer/Cancer Hospital, Chinese Academy of Medical Sciences and Peking Union Medical College, Beijing 100021, China (e-mail: dai_jianrong@ @icams.ac.cn). 
for RSSI fluctuation and to adapt to different environments, we propose an optimization method combining RSSI and PDR data to estimate the distance between smart devices. The PDR may provide the high accuracy of walking distance and direction, which is used to compensate for the effects of interference on the RSSI. Moreover, the parameters of the log-distance path loss model optimized by particle swarm optimization (PSO) are used to dynamically fit the complex electromagnetic environment.

In summary, this paper has the following contributions:

- An optimization method is developed to estimate the distance between smart devices by combining RSSI and PDR Data. The method optimizes the parameters of the RSSIdistance model and the relative locations of each pair of devices simultaneously.

- The method does not require a time-consuming fingerprint database buildup for an environment, and thus, it has high feasibility and low complexity.

- The performance of the method is tested in outdoor and indoor environments. The results show better accuracy of distance estimation than the RSSI-only method.

The rest of this paper is organized as follows. Section 2 introduces the related works. Section 3 describes the proposed distance estimation method. Section 4 shows the experimental setup and procedure, as well as the evaluation results for several scenarios in outdoor and indoor environments. Finally, Section 5 concludes this paper and presents some future directions.

\section{RELATED WORK}

\section{A. Log-Distance Path Loss Model}

The RSSI-only method generally uses the log-distance path loss (LDPL) model [11]. It is expressed as

$$
R S S I=A-10 * n * \lg \left(\frac{d}{d_{0}}\right)+\varepsilon
$$

where $d$ is the distance between the transmitter and the receiver, and $n$ is the path loss exponent which varies depending upon the radio propagation environment. $A$ is the RSSI at the reference distance $d_{0}$ from the transmitter. $\varepsilon$ is a Gaussian distribution random variable with mean zero.

For convenience, $d_{0}$ is assigned as 1 meter, and $\varepsilon$ has a mean value of zero. Then the LDPL model can be obtained with

$$
R S S I=A-10 * n * \lg (d)
$$

where $A$ is the average measured RSSI when the receiver is placed 1 meter away from the transmitter. To calibrate the parameters $A$ and $n$, the RSSI-only method requires utilizing the least-squares algorithm to fit the LDPL model, by measuring the RSSI values at different distances in advance [22]. Since these two parameters need to be obtained before distance measurement, for an unknown situation, distance estimation may have inaccuracies because it uses parameters not fitted to the environment.

Recent studies try to dynamically adjust the parameters of the RSSI-distance model by using a neural network. Nonetheless, it is difficult to implement these methods into real-world applications, especially for real-time Covid-19 contact tracing, because of computing with high complexity, requiring a large amount of calibrated data and training the neural network for the known scenario before distance estimation [15], [23].

In this study, the LDPL model is optimized by particle swarm optimization without training. The RSSI-distance between two devices, $d_{r}$, is achieved during the optimization process, which is also a part of the optimization objective function. A detailed description of the optimization process is provided in section 3.

\section{B. Pedestrian Dead Reckoning (PDR)}

The PDR is a relative location method based on walking data of pedestrians, which can navigate with low-cost devices (e.g. the inertial sensors available in most smartphones, such as accelerometer, magnetometer, and gyro sensor) [24], [25]. It is comprised of three main parts: (i) pedestrian's start location, (ii) walking distance, and (iii) walking direction, as depicted in Fig. 1, where $\mathrm{E}$ and $\mathrm{N}$ represent the East and North directions, respectively.

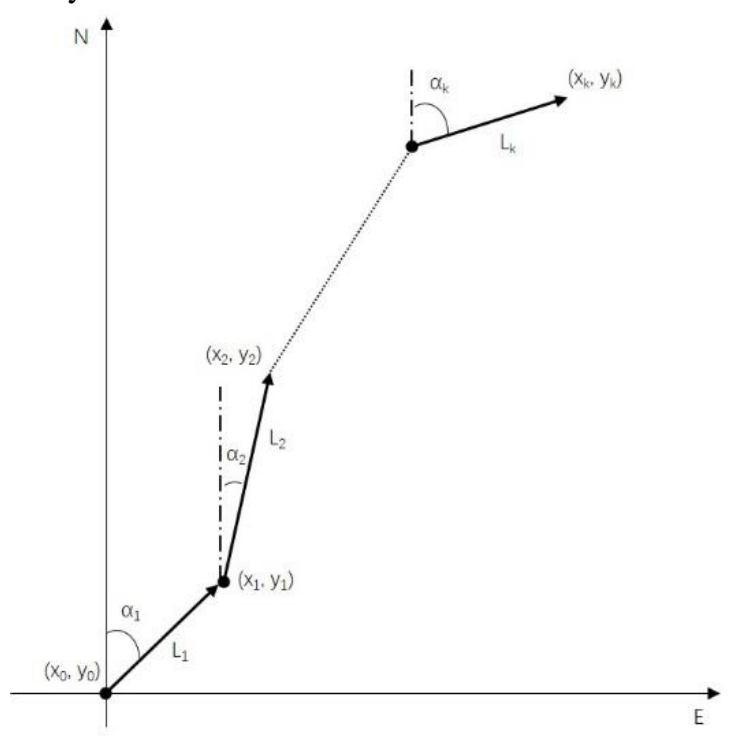

Fig. 1. Schematic diagram of pedestrian dead reckoning (PDR) method for distance estimation.

The formula for the PDR can be defined as in equation (3):

$$
\left\{\begin{array}{l}
x_{k}=x_{0}+\sum_{i=1}^{k} L_{i} \sin \left(\alpha_{i}\right) \\
y_{k}=y_{0}+\sum_{i=1}^{k} L_{i} \cos \left(\alpha_{i}\right)
\end{array}\right.
$$

Here, $\alpha_{i}$ and $L_{i}$ represent the walking direction (the angle between forwarding direction and North) and the walking distance from the $(i-1)$ th sampling point to the $i$ th, respectively. The coordinates can be calculated by equation (3) if $\alpha_{i}$ and $L_{i}$ are obtained. Since the properties of the RSSIbased distance estimation method and PDR-based localization method are complementary, so the combination of these two methods would be beneficial to improve the estimation accuracy

In this study, the PDR-distance between two devices, $d_{p}$, is obtained from the coordinates of the two devices' locations. $d_{p}$ is also a part of the optimization objective function. 


\section{MATERIALS AND METHODS}

For a pair of two smart devices, device \#1 and device \#2, suppose that there are $m$ sampling points during their recorded contacting paths, as Fig. 2 shows.

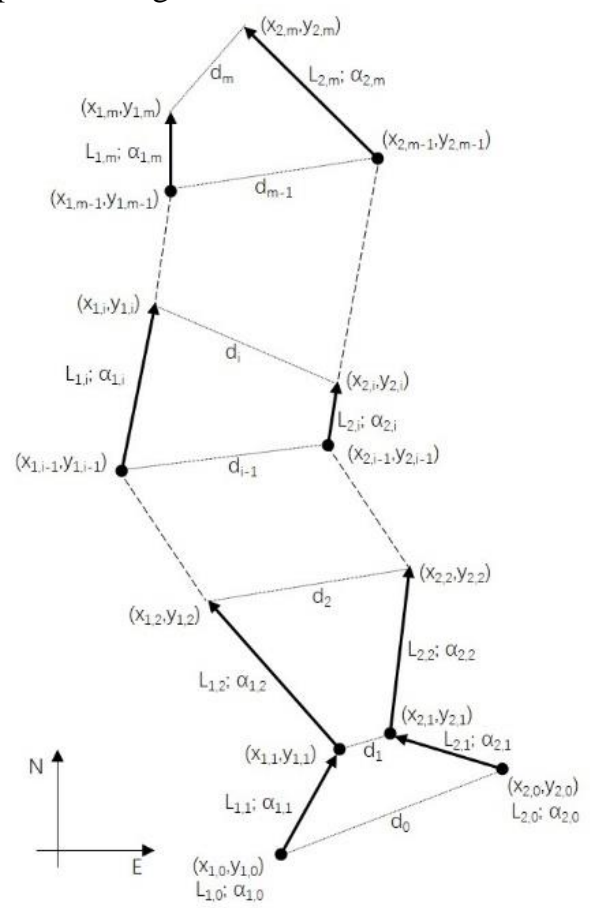

Fig. 2. Schematic diagram of the recorded contacting path from device \#1 and device \#2.

In Fig. 2, $\left(x_{i}, y_{i}\right)$ denotes the coordinates of the device at the $i$ th sampling point; $L_{i}$ denotes the walking distance from the $i-1$ th sampling point to the $i$ th; $\alpha_{i}$ denotes the walking direction, $0 \leq \alpha<360 ; d_{i}$ denotes the real distance between two devices at the $i$ th sampling point. The subscript 1 and 2 of $x, y, L$, and $\alpha$ represent device \#1 and device \#2, respectively. The subscript 0 represents the start point of this recorded contacting path. Additionally, at each sampling point, the receiver will record the RSSI, which can be denoted as $R_{i}$ for the $i$ th time point. Matrix $Q$ is defined to contain all of the above data, that is,

$$
Q=\left[\begin{array}{ccccc}
L_{1,0} & \alpha_{1,0} & L_{2,0} & \alpha_{2,0} & R_{0} \\
& & \vdots & & \\
L_{1, i} & \alpha_{1, i} & L_{2, i} & \alpha_{2, i} & R_{i} \\
& & \vdots & & \\
L_{1, m} & \alpha_{1, m} & L_{2, m} & \alpha_{2, m} & R_{m}
\end{array}\right]
$$

For simplicity's sake, the start point of device \#1 is defined as the origin of the coordinate system, and the start point of device $\# 2$ is defined as $(\Delta x, \Delta y)$ in this coordinate system, that is,

$$
\begin{gathered}
x_{1,0}=0, y_{1,0}=0 \\
x_{2,0}=\Delta x, y_{2,0}=\Delta y
\end{gathered}
$$

According to Section 2, the distance between the two devices can be calculated from both the RSSI-only method and the PDR method. These two methods will be described in the following subsections.

\section{A. Distance Estimation by Using RSSI-Only Method}

For the RSSI-only method, according to equation (2), the distance at the $k$ th sampling point can be calculated by the following equation:

$$
d_{r, k}=10^{\left[\frac{\left(A-R_{k}\right)}{10 * n}\right]}
$$

Consequently, the distance estimation of RSSI-only method,

$$
d_{r}=\left(d_{r, 0}, d_{r, 1}, \ldots, d_{r, k}, \ldots, d_{r, m}\right)
$$

can be written as a function of $R, A$ and $n$, that is,

$$
d_{r}=g(R, A, n)
$$

it can also be written as

$$
d_{r}=g(Q, A, n),
$$

where $Q$ is the known data; $A$ and $n$ are the optimization variables.

\section{B. Distance Estimation by Using PDR Method}

According to equation (3) and (5), the coordinates of device $\# 1$ at the $k$ th sampling point are

$$
\begin{aligned}
& x_{1, k}=x_{1,0}+\sum_{i=1}^{k} L_{1, i} \sin \left(\alpha_{1, i}\right)=\sum_{i=1}^{k} L_{1, i} \sin \left(\alpha_{1, i}\right) \\
& y_{1, k}=y_{1,0}+\sum_{i=1}^{k} L_{1, i} \cos \left(\alpha_{1, i}\right)=\sum_{i=1}^{k} L_{1, i} \cos \left(\alpha_{1, i}\right)
\end{aligned}
$$

Similarly, according to equation (3) and (6), the coordinates of device \#2 at the $k$ th sampling point are

$$
\begin{aligned}
& x_{2, k}=x_{2,0}+\sum_{i=1}^{k} L_{2, i} \sin \left(\alpha_{2, i}\right)=\Delta x+\sum_{i=1}^{k} L_{2, i} \sin \left(\alpha_{2, i}\right) \\
& y_{2, k}=y_{2,0}+\sum_{i=1}^{k} L_{2, i} \cos \left(\alpha_{2, i}\right)=\Delta y+\sum_{i=1}^{k} L_{2, i} \cos \left(\alpha_{2, i}\right)
\end{aligned}
$$

So, for the PDR method, the distance at the $k$ th sampling point can be calculated by the following equation:

$$
\begin{aligned}
& d_{p, k} \\
& =\left(\begin{array}{c}
{\left[\left(\Delta x+\sum_{i=1}^{k} L_{2, i} \sin \left(\alpha_{2, i}\right)\right)-\sum_{i=1}^{k} L_{1, i} \sin \left(\alpha_{1, i}\right)\right]^{2}} \\
+\left[\left(\Delta y+\sum_{i=1}^{k} L_{2, i} \cos \left(\alpha_{2, i}\right)\right)-\sum_{i=1}^{k} L_{1, i} \cos \left(\alpha_{1, i}\right)\right]^{\frac{1}{2}}
\end{array}\right)^{2}
\end{aligned}
$$

Consequently, the distance estimation of the PDR method,

$$
d_{p}=\left(d_{p, 0}, d_{p, 1}, \ldots, d_{p, k}, \ldots, d_{p, m}\right)
$$

can be written as a function of $L, \alpha, \Delta x$ and $\Delta y$, that is,

$$
d_{p}=h(L, \alpha, \Delta x, \Delta y)
$$

it can also be written as 


$$
d_{p}=h(Q, \Delta x, \Delta y)
$$

where $Q$ is the known data; $\Delta x$ and $\Delta y$ are the optimization variables.

\section{Proposed Optimization Method}

Based on the equation (10) and (18), the objective function of the distance estimation optimization problem is proposed:

$$
\min f\left(d_{r}, d_{p}\right)
$$

that is,

$$
\min _{A, n, \Delta x, \Delta y} f(g(Q, A, n), h(Q, \Delta x, \Delta y))
$$

The function $f$ is defined as

$$
f\left(d_{r}, d_{p}\right)=\sum_{i=1}^{m}\left(d_{r, i}-d_{p, i}\right)^{2}
$$

So, the objective function can be written as

$$
\min \sum_{i=1}^{m}\left(d_{r, i}-d_{p, i}\right)^{2}
$$

that is,

$$
\min _{A, n, \Delta x, \Delta y} \sum_{i=1}^{m}(g(Q, A, n)-h(Q, \Delta x, \Delta y))^{2}
$$

where $Q$ is the known data; $A, n, \Delta x$ and $\Delta y$ are the optimization variables.

The constraints of this optimization problem include three items:

(1) The domain of the model parameters $A$ and $n$.

For common smartphones, the value of $A$ is in the range of $80 \mathrm{dBm}$ and $-40 \mathrm{dBm} . n$ is the path loss exponent that varies depending upon the radio propagation environment. T. S. Rappaport gives typical values for $n$ in outdoor and indoor environments. The minimal value of $n$ is 2 for free space, while the maximum can be set to 6 for obstructed building. The constraints are written as

$$
\begin{gathered}
-40-A \geq 0 \\
A+80 \geq 0 \\
n-2 \geq 0 \\
6-n \geq 0
\end{gathered}
$$

(2) The domain of the relative coordinates of device \#2 start point.

Due to the range of Bluetooth low-energy (BLE) is about 100 $\mathrm{m}$, the minimal relative coordinate value of device \#2 start point can be set to $-100 \mathrm{~m}$, and the maximum can be set to $100 \mathrm{~m}$. The constraints are written as

$$
\begin{aligned}
& \Delta x+100 \geq 0 \\
& 100-\Delta x \geq 0 \\
& \Delta y+100 \geq 0 \\
& 100-\Delta y \geq 0
\end{aligned}
$$

(3) The effective measurable range of the proposed distance estimation method.
The effective measurable range depends on the application scenario. For Covid-19 contact tracing apps, the minimal effective distance can be set to $0.1 \mathrm{~m}$, and the maximum can be set to $10 \mathrm{~m}$. The constraints are written as

$$
\begin{gathered}
g(Q, A, n)-0.1 \geq 0 \\
10-g(Q, A, n) \geq 0 \\
h(Q, \Delta x, \Delta y)-0.1 \geq 0 \\
10-h(Q, \Delta x, \Delta y) \geq 0
\end{gathered}
$$

The above optimization problem is solved by the particle swarm optimization (PSO) algorithm. The PSO algorithm first creates initial particles and then assigns initial velocities to them. It evaluates the objective function (fitness) of each particle position and determines the best function value and the best position. It selects a new velocity based on the current velocity, the individual best position of the particle (pbest), and the best position among all the population (gbest). Then, it iteratively updates the particle position and velocity (the new position is the old position plus the velocity, and keeps the particle within the boundary). The algorithm iterates until reaches the stopping criterion. The following two equations illustrate the searching process:

$$
\begin{gathered}
v_{i, j+1}=w * v_{i, j}+c_{1} * u_{1} *\left(\text { pbest }_{i}-x_{i, j}\right) \\
+c_{2} * u_{2} *\left(\text { gbest }_{j}-x_{i, j}\right) \\
x_{i, j+1}=v_{i, j}+x_{i, j}
\end{gathered}
$$

where $i$ denotes the particle index and $j$ denotes the iteration index. $x_{i, j}$ and $v_{i, j}$ represent the position and velocity of particle $i$ in iteration $j$, respectively. $u_{1}$ and $u_{2}$ are random numbers uniformly distributed in the interval $(0,1) \cdot c_{1}$ and $c_{2}$ are positive constants, where $c_{1}$ is the self-adjustment weight and $c_{2}$ is the global adjustment weight. $w$ presents inertia weight. In this study, the value of $w$ was constantly reduced from 0.8 to 0.4 during the searching process. $c_{1}$ and $c_{2}$ were set to 0.4 and 0.6 , respectively.

\section{THE EXPERIMENTAL SETUP AND RESUltS}

\section{A. Outdoor Experiments with Participants}

We placed 13 sampling points $(\mathrm{A} \sim \mathrm{M})$ on outdoor park ground, as the following Fig. 3 shows. Two participants walked from the start point to the endpoint with smartphones, following the designed path (detailed description in Table. I). The first participant held device \#1 (iPhone XS) as a transmitter; the second participant held device \#2 (Samsung Note10) as a receiver. 


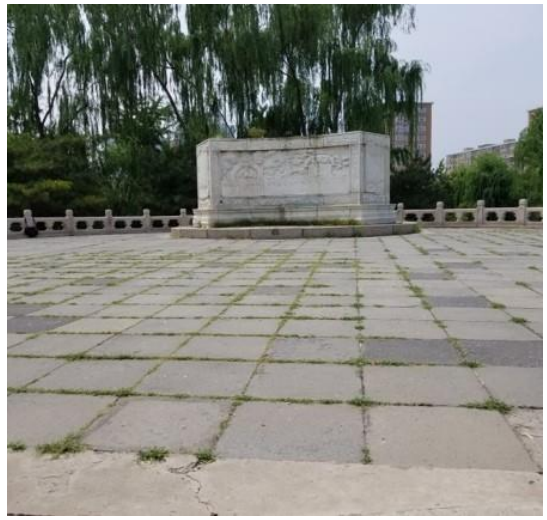

(a)

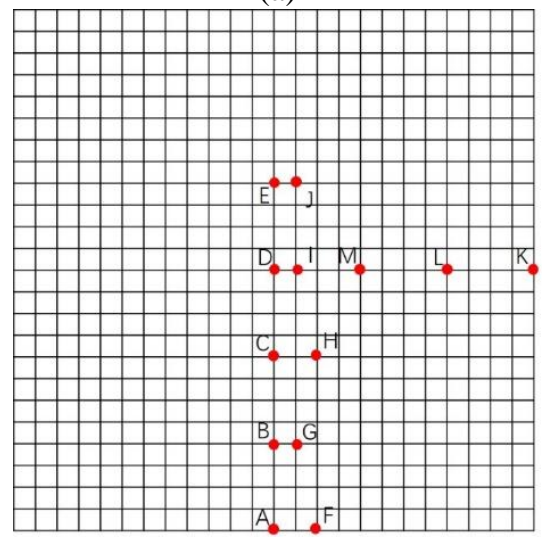

(b)

Fig. 3. The experimental setup in the outdoor environment: (a) The picture of the park ground; (b) The schematic diagram of the experimental setup for the park ground. The side length of the grid is about 0.5 meters, and the 13 sampling points $(\mathrm{A} \sim \mathrm{M})$ were located at the vertex of the grid.

For the RSSI-only method, the RSSI-distance model's parameter $A$ was set to $65 \mathrm{dBm}$ and $n$ was 3.3 , after a typical calibration procedure. For the proposed method, the domain of $A$ was between -80 and $-40 \mathrm{dBm}$, and the domain of $n$ was between 2 and 6 . These values and domains of parameters were also used for indoor experiments. To reduce the effects of signal fluctuation, at each sampling point, we measured RSSI for one minute. The median of sampled RSSI was considered as the RSSI value for our experiments. The distance between two smartphones was estimated by using the RSSI-only method and the proposed method, respectively. Then, we measured the real distance to evaluate the performance of these two methods. The real distance, the estimation errors of the RSSI-only method, and the proposed method were shown in Table. I.

We can see that the estimation results of the RSSI-only method were still average even when the parameter calibration was used. The mean value was $0.43 \mathrm{~m}$, the root mean square error (RMSE) was $0.96 \mathrm{~m}$, the mean absolute error (MAE) was $0.57 \mathrm{~m}$, and the maximum error was $3.45 \mathrm{~m}$. The proposed method was able to reduce the estimation error: the mean was $0.10 \mathrm{~m}$, the RMSE was $0.76 \mathrm{~m}$, the MAE was $0.51 \mathrm{~m}$, and the maximum error was $2.23 \mathrm{~m}$. The results were compared with the paired-samples $t$-test using SPSS statistical software version 26.0. The statistical results indicated that there was a significant difference between the two methods, $P$-value $<0.00001$.

TABLE. I

THE RESUltS OF THE OUTDOOR EXPERIMENTS (ALL IN METERS)

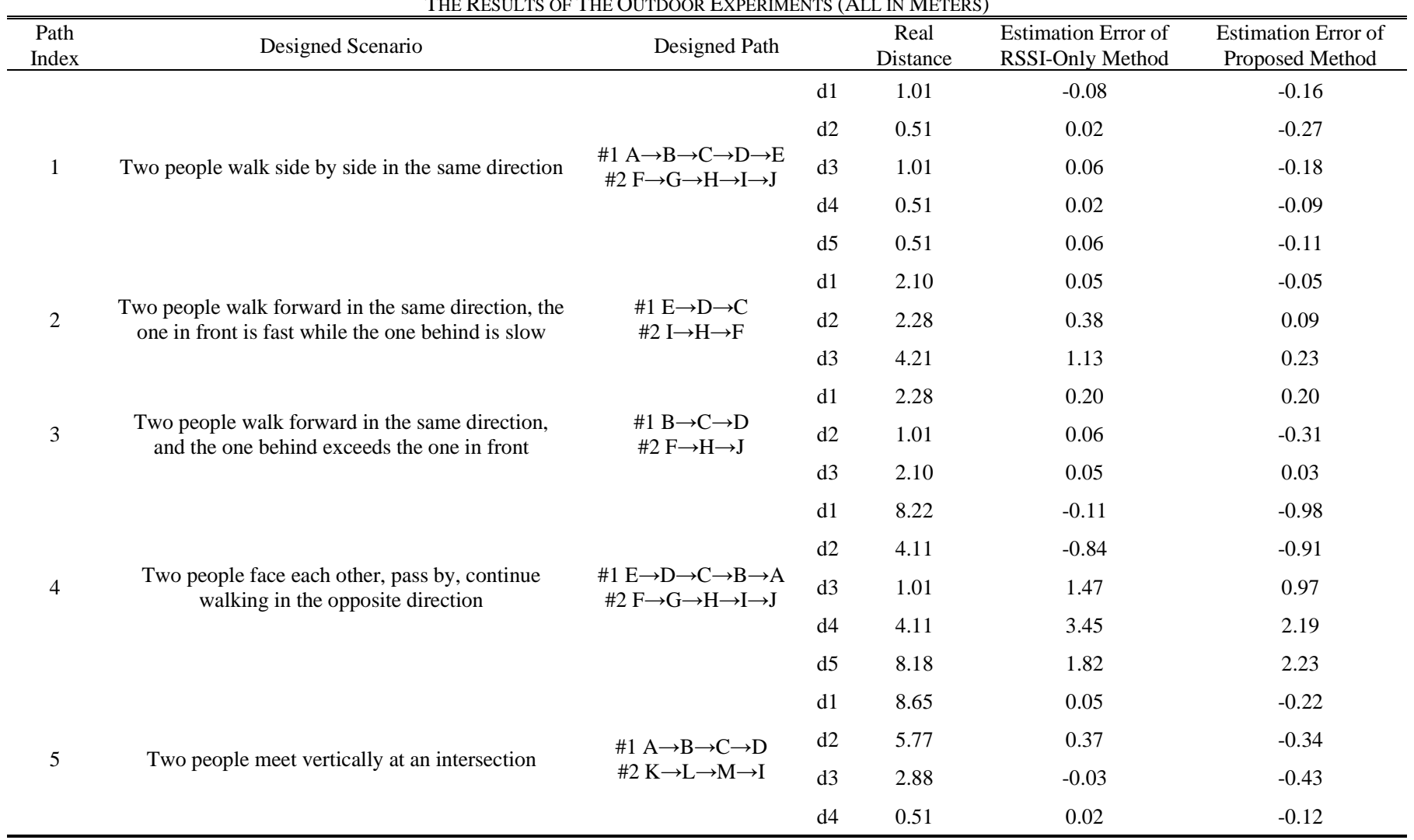




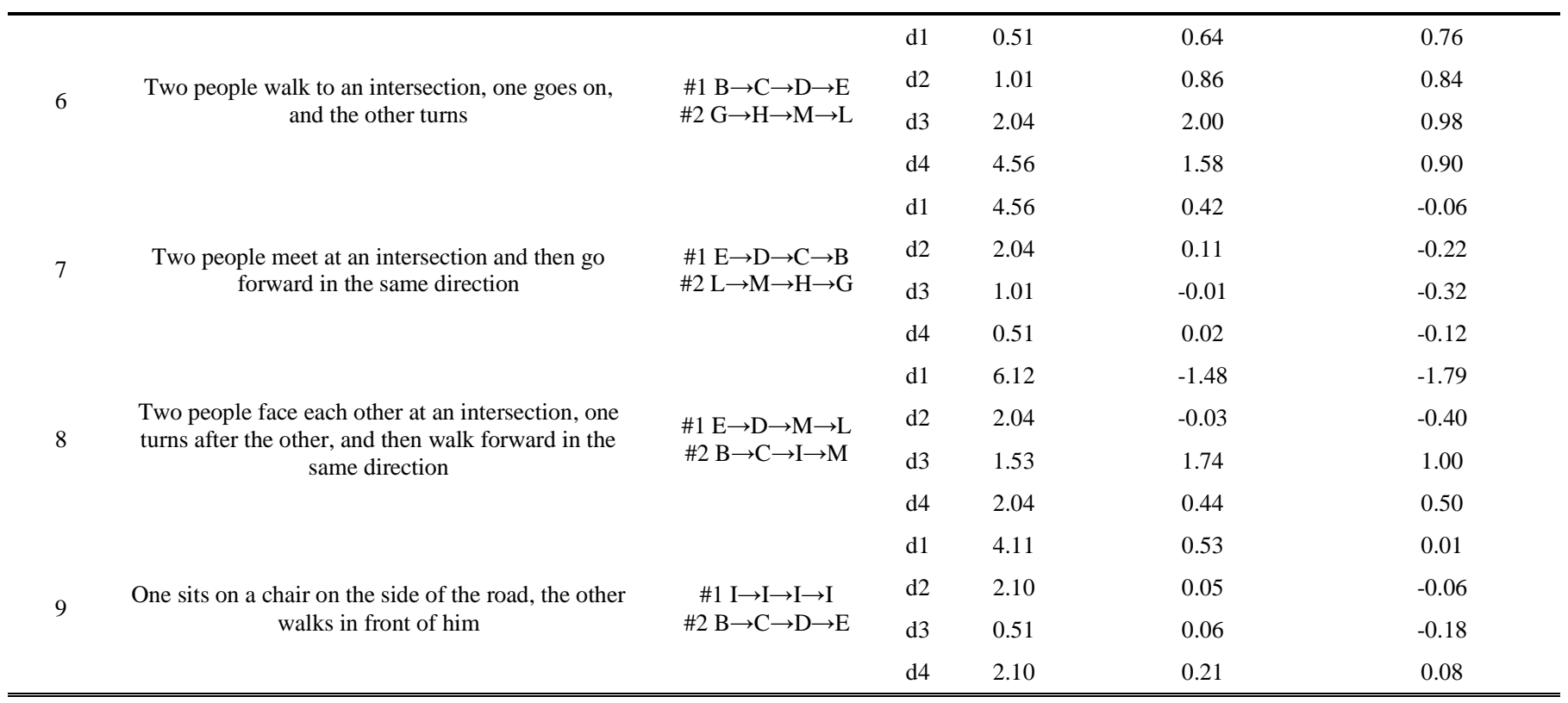

\section{B. Indoor Experiments with Participants}

We placed 16 sampling points (A I, O, M1 M6) on an indoor meeting room ground, as the following Fig. 4 shows. The first participant held iPhone XS (transmitter) at point I, the second participant walked from the start point to the endpoint with Samsung Note10 (receiver), following the designed path (detailed description in Table. II).

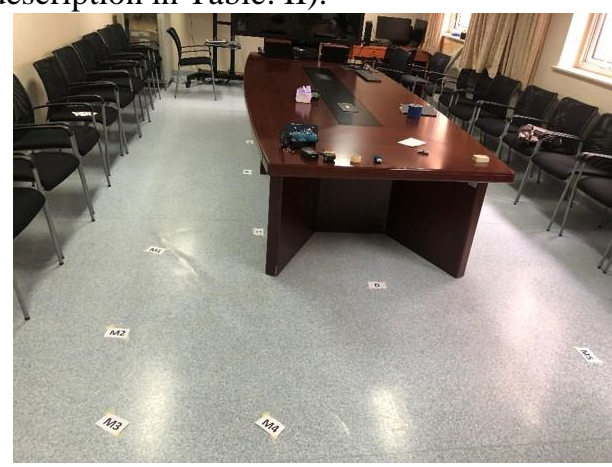

(a)

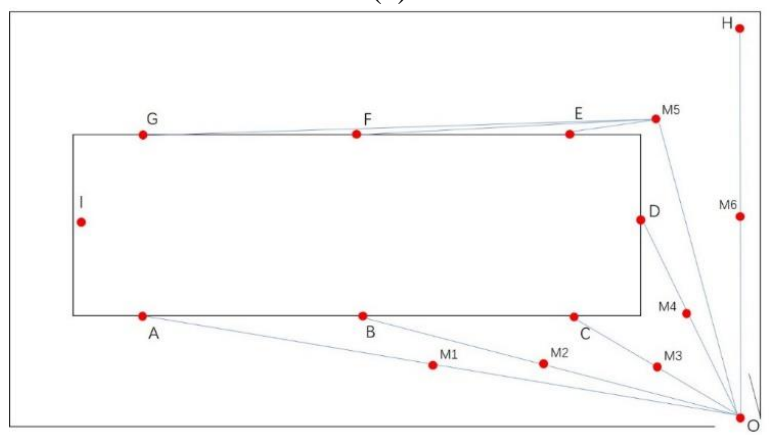

(b)

Fig. 4 The experimental setup in the indoor environment: (a) The picture of the meeting room. There was a desk in the center of a meeting room. Some obstacles were on the desk, such as computers, books, and bottles of water. Point $\mathrm{O}$ was the start point for all designed paths, which are located at the door; (b) The schematic diagram of the experimental setup for the meeting room.

Similarly, as the outdoor experiment, the distance between two smartphones was estimated by using the RSSI-only method and the proposed method, respectively. Then, we measured the real distance to evaluate the performance of these two methods. The real distance, the estimation errors of the RSSI-only method, and the proposed method were listed in Table. II.

We can see that the estimation results of the RSSI-only method were poor when the parameter $A$ and $n$ used the fixed value. The mean value was $-2.88 \mathrm{~m}$, the RMSE was $3.24 \mathrm{~m}$, the MAE was $2.89 \mathrm{~m}$, and the maximum error was $4.49 \mathrm{~m}$. The proposed method was able to effectively reduce the estimation error: the mean was $-0.31 \mathrm{~m}$, the RMSE was $1.21 \mathrm{~m}$, the MAE was $1.02 \mathrm{~m}$, and the maximum error was $2.15 \mathrm{~m}$. The pairedsamples $t$-test results indicated that there was a significant difference between the two methods, $P$-value $<0.00001$.

The results show that the proposed method has better distance estimation accuracy than the RSSI-only method, and demonstrate its feasibility through real-world evaluations.

TABLE. II

THE RESUlts OF THE INDOOR EXPERIMENTS (ALL IN METERS) 


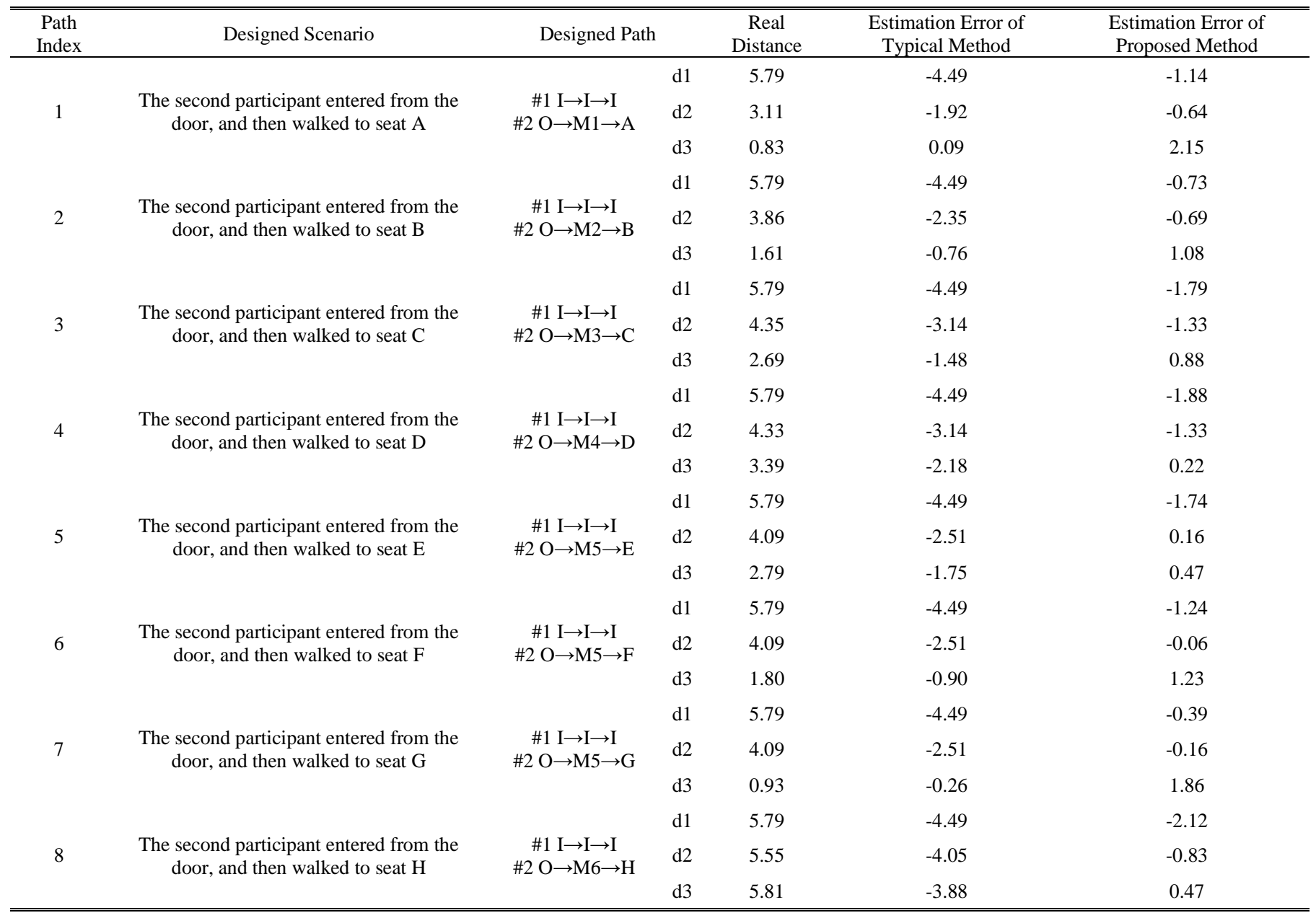

\section{CONClusions AND Future Work}

This paper presents a novel optimization method to estimate the distance between smart devices based on RSSI and PDR data by using particle swarm optimization. The PDR may provide the high accuracy of walking distance and direction, which is used to compensate for the effects of interference on the RSSI. Moreover, the parameters of the log-distance path loss model optimized by PSO are used to dynamically fit the complex electromagnetic environment. The performance of the method is tested in outdoor and indoor environments. The results show better accuracy of distance estimation than the RSSI-only method and demonstrate its high feasibility and low complexity. Therefore, the proposed method can be further integrated into the positioning systems for wireless sensor networks and proximity alert apps for Covid- 19 contact tracing. We hope that more researchers or research institutions will be interested in further testing the effectiveness of this method.

\section{REFERENCES}

P. C. Ng, J. She, K. E. Jeon, and M. Baldauf, "When Smart Devices Interact With Pervasive Screens," ACM Transactions on Multimedia Computing, Communications, and Applications, vol. 13, no. 4, pp. 1-23, 2017, doi: 10.1145/3115933.

[2] A. H. Sayed, A. Tarighat, and N. Khajehnouri, "Network-based wireless location: challenges faced in developing techniques for accurate wireless location information," IEEE Signal Processing
Magazine, vol. 22, no. 4, pp. 24-40, 2005, doi: 10.1109/msp.2005.1458275.

[3] M. Passafiume, S. Maddio, and A. Cidronali, "An Improved Approach for RSSI-Based only Calibration-Free Real-Time Indoor Localization on IEEE 802.11 and 802.15.4 Wireless Networks," Sensors (Basel), vol. 17, no. 4, p. 717, 2017, doi: 10.3390/s17040717.

[4] K. H. Grantz et al., "The use of mobile phone data to inform analysis of COVID-19 pandemic epidemiology," Nat Commun, vol. 11, no. 1, p. 4961, Sep 30 2020, doi: 10.1038/s41467-020-18190-5.

[5] L. Ferretti et al., "Quantifying SARS-CoV-2 transmission suggests epidemic control with digital contact tracing," Science, vol. 368, no. 6491, May 8 2020, doi: 10.1126/science.abb6936.

[6] A. Bilinski, F. Mostashari, and J. A. Salomon, "Modeling Contact Tracing Strategies for COVID-19 in the Context of Relaxed Physical Distancing Measures," JAMA Network Open, vol. 3, no. 8, pp. e2019217-e2019217, 2020, doi: 10.1001/jamanetworkopen.2020.19217.

[7] O. G. Adewumi, K. Djouani, and A. M. Kurien, "RSSI based indoor and outdoor distance estimation for localization in WSN," ed: IEEE, 2013, pp. 1534-1539.

[8] Y. Zhuang, J. Yang, Y. Li, L. Qi, and N. El-Sheimy, "SmartphoneBased Indoor Localization with Bluetooth Low Energy Beacons," Sensors (Basel), vol. 16, no. 5, p. 596, 2016, doi: 10.3390/s16050596.

[9] S. Mazuelas et al., "Robust Indoor Positioning Provided by RealTime RSSI Values in Unmodified WLAN Networks," IEEE Journal of Selected Topics in Signal Processing, vol. 3, no. 5, pp. 821-831, 2009, doi: 10.1109/jstsp.2009.2029191.

[10] B. Wang, S. Zhou, W. Liu, and Y. Mo, "Indoor Localization Based on Curve Fitting and Location Search Using Received Signal Strength," IEEE Transactions on Industrial Electronics, vol. 62, no. 1, pp. 572-582, 2015, doi: 10.1109/tie.2014.2327595. 
J. B. Andersen, T. S. Rappaport, and S. Yoshida, "Propagation measurements and models for wireless communications channels," IEEE Communications Magazine, vol. 33, no. 1, pp. 42-49, 1995, doi: $10.1109 / 35.339880$.

[12] P. Chuan Chin, O. Pei Cheng, L. Boon Giin, and C. Wan-Young, "Analysis of path loss exponent error in ranging and localization of wireless sensor network," ed. Stevenage, UK: Stevenage, UK: IET, 2014, p. 17.

[13] J. Jung, D. Kang, and C. Bae, "Distance Estimation of Smart Device using Bluetooth," in the Eighth International Conference on Systems and Networks Communications, Venice, Italy, 27 October-1 November 2013, 2013, pp. 13-18.

[14] A. Reed, "Apple and Google launch 'exposure notification' tool to aid contact tracing," The Washington post, 2020.

[15] D. J. Leith and S. Farrell, "Measurement-based evaluation of Google/Apple Exposure Notification API for proximity detection in a light-rail tram," PLoS One, vol. 15, no. 9, p. e0239943, 2020, doi: 10.1371/journal.pone.0239943

[16] R. Liu, C. Yuen, T.-N. Do, and U. X. Tan, "Fusing Similarity-Based Sequence and Dead Reckoning for Indoor Positioning Without Training," IEEE Sensors Journal, vol. 17, no. 13, pp. 4197-4207, 2017, doi: 10.1109/jsen.2017.2706303.

[17] K. Lee, Y. Nam, and S. D. Min, "An indoor localization solution using Bluetooth RSSI and multiple sensors on a smartphone," Multimedia Tools and Applications, vol. 77, no. 10, pp. 1263512654, 2017, doi: 10.1007/s11042-017-4908-2.

[18] S. Tiwari and V. k. Jain, "HILS: hybrid indoor localisation system using Wi-Fi received signal strength and inertial sensor's measurements of smart-phone," IET Communications, vol. 13, no. 11, pp. 1595-1606, 2019, doi: 10.1049/iet-com.2018.5845.

[19] K. Han, H. Xing, Z. Deng, and Y. Du, "A RSSI/PDR-Based Probabilistic Position Selection Algorithm with NLOS Identification for Indoor Localisation," ISPRS International Journal of Geo-Information, vol. 7, no. 6, 2018, doi: 10.3390/ijgi7060232.

[20] X.-W. Shi and H.-Q. Zhang, "Research on indoor location technology based on back propagation neural network and Taylor series," ed: IEEE, 2012, pp. 1886-1890.

[21] G. Li, E. Geng, Z. Ye, Y. Xu, J. Lin, and Y. Pang, "Indoor Positioning Algorithm Based on the Improved RSSI Distance Model," Sensors (Basel), vol. 18, no. 9, Aug 27 2018, doi: $10.3390 / \mathrm{s} 18092820$.

[22] K. Sung, D. K. Lee, and H. Kim, "Indoor Pedestrian Localization Using iBeacon and Improved Kalman Filter," Sensors (Basel), vol. 18, no. 6, May 26 2018, doi: 10.3390/s18061722.

[23] S. Shankar et al., "Proximity Sensing: Modeling and Understanding Noisy RSSI-BLE Signals and Other Mobile Sensor Data for Digital Contact Tracing," 2020.

[24] D. Kamisaka, S. Muramatsu, T. Iwamoto, and H. Yokoyama, "Design and Implementation of Pedestrian Dead Reckoning System on a Mobile Phone," IEICE transactions on information and systems, vol. E94-D, no. 6, pp. 1137-1146, 2011, doi: 10.1587/transinf.E94.D.1137.

[25] J. Qian, L. Pei, J. Ma, R. Ying, and P. Liu, "Vector graph assisted pedestrian dead reckoning using an unconstrained smartphone," Sensors (Basel), vol. 15, no. 3, pp. 5032-5057, 2015, doi: $10.3390 / \mathrm{s} 150305032$.

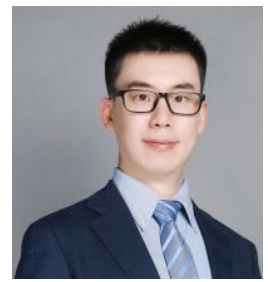

Bo Zhao was born in Beijing, China in 1986. He received a bachelor's degree in engineering physics from Tsinghua University in 2008 and got a Ph.D. degree in medical physics from Peking Union Medical College in 2013. He is currently working in the Department of Engineering Physics, Tsinghua University and Key Laboratory of Particle \& Radiation Imaging, Ministry of Education (Tsinghua University), as a research associate professor. His research interests are in the area of optimization algorithms and artificial intelligence. He has published two research articles about optimization algorithm development with considering marker visibility for segmental and dynamic intensity-modulated radiation therapy, and two other articles about dose prediction and auto-planning with artificial intelligence methods.

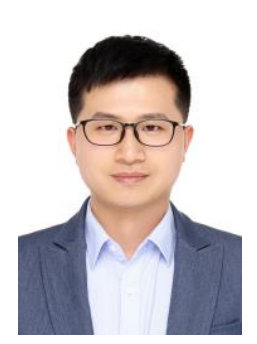

Chao Zheng was born in Zhejiang, China in 1988. He received a bachelor's degree in physics from Zhejiang University in 2010 and got a master's degree in medical physics from Peking Union Medical College in 2013. He is currently working in Haichuang Era Medical Technology Ltd as chief executive officer. He focuses on the research and development of artificial intelligence and the informatization of radiation oncology.

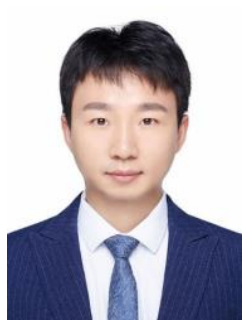

Xinxin Ren was born in Guizhou, China in 1990. He received a bachelor's degree in engineering physics and a master's degree in Oncology from Tsinghua University, Beijing, China, in 2012 and 2015. He is currently working in Haichuang Era Medical Technology Ltd as chief technology officer. His main research interests are the application of artificial intelligence and data analysis in medical science.

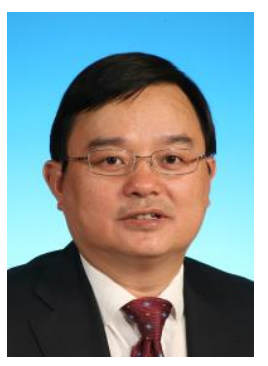

Jianrong Dai was born in Hunan, China, in 1966. He received a bachelor's degree in nuclear physics from Peking University in 1988 and got a Ph.D. degree in medical physics from Peking Union Medical College in 1996. He is currently working in the Department of Radiation Oncology, National Cancer Center/National Clinical Research Center for Cancer/Cancer Hospital, Chinese Academy of Medical Sciences, and Peking Union Medical College, as vice-chairman. Dr. Dai's research is primarily related to developing, improving, and implementing new methods, algorithms, or techniques for radiotherapy. He has over 130 peer-reviewed publications. 